\title{
中性粒细胞在哮喘中的地位和作用
}

\author{
陈 菲, 虞 敏, 钟永红, 华 雯, 黄华琼 \\ 浙江大学医学院附属第二医院呼吸与危重症医学科, 浙江杭州 310009
}

[摘要] 支气管哮喘是由多种细胞包括气道的炎性细胞和结构细胞及细胞组分 参与的气道慢性炎症性疾病, 其特征为气道炎症、气道高反应性、可逆性气道阻 塞和气道重塑。以往哮喘被认为主要由 2 型炎症因子驱动, Th2 细胞分泌IL-4、IL-5 和IL-13, 引起气道嗜酸性粒细胞炎症。随着研究的深入, 发现中性粒细胞与哮喘炎 症过程也存在密切关系。哮喘患者气道中的中性粒细胞在趋化增加的同时调亡下 降, 从而导致中性粒细胞数量增多。中性粒细胞比嗜酸性粒细胞更早到达, 通过产 生弹性蛋白酶、䯣过氧化物酶、中性粒细胞胞外诱捕网、趋化因子和细胞因子 等, 参与哮喘的发生及发展过程。针对这些效应分子的拮抗剂如抗 IL-8 受体抗 体、抗 IL-17 抗体, DNA 酶显示出对中性粒细胞哮喘的治疗作用, 但应用于临床还 需要更多的实验数据支持。本文主要就中性粒细胞在哮喘中的地位和作用作一 综述。

[关键词] 中性粒细胞; 哮喘; 中性粒细胞哮喘; 治疗; 综述 [中图分类号] R562.1 [文献标志码 ] A

\section{The role of neutrophils in asthma}

CHEN Fei, YU Min, ZHONG Yonghong, HUA Wen, HUANG Huaqiong(Department of Respiratory and Critical Care Medicine,the Second Affiliated Hospital,Zhejiang University School of Medicine, Hangzhou 310009, China)

Corresponding author:HUANG Huaqiong,_E-mail:zr_hhq@zju.edu.cn, https://orcid.org/ 0000-0001-9017-7835

\begin{abstract}
[Abstract ] Bronchial asthma is a chronic respiratory disease, characterized by airway inflammation, airway hyperresponsiveness, reversible airway obstruction and airway remodeling, in which a variety of cells including airway inflammatory cells and structural cells are involved. Previous studies have shown that asthma is mainly driven by Th2 cytokines IL-4, IL-5, and IL-13, leading to airway eosinophil inflammation. With further research, however, it has been found that neutrophils are also closely related to asthma.
\end{abstract}

\section{收稿日期: 2020-09-14接受日期:2020-12-01}

基金项目:国家重点研发计划(2017YFC131060); 浙江省自然科学基金(LQ18H010002)

第一作者:陈 菲,硕士研究生,主要从事呼吸系统慢性疾病研究; E-mail:chenfei66@zju. edu. cn; https://orcid. org/ 0000-0001-6017-2785

通信作者:黄华琼,副主任医师,硕士生导师,主要从事慢性气道疾病防治和机制研究; E-mail:zr_hhq@zju. edu. cn; https ://orcid. org/0000-0001-9017-7835 
Numbers of neutrophils are elevated in airway through increased chemotaxis and decreased apoptosis, which is earlier than eosinophils, leading to airway neutrophilic inflammation. Neutrophils can produce elastase,myeloperoxidase, neutrophil extracellular traps, chemokines and cytokines, participating in the occurrence and development of asthma. The antagonists against these molecules, such as anti-IL-8 receptor antibody, anti-IL-17 antibody, and DNase, have shown positive effects on neutrophilic asthma, but further studies are needed to support their clinical application. This article mainly reviews the role of neutrophils in asthma and related mechanisms.

[Key words ] Neutrophils; Asthma; Neutrophilic asthma; Therapy; Review

[ J Zhejiang Univ (Med Sci), 2021, 50(1): 123-130. ]

[缩略语] 白细胞介素(interleukin,IL); 粒细胞-巨噬细胞集落刺激因子 (granulocytemacrophage colony-stimulating factor, GM-CSF); 肿瘤坏死因子 (tumor necrosis factor, TNF); 白三烯 B4 (leukotriene B4,LTB4); 中性粒细胞胞外诱捕网 (neutrophil extracellular traps, NET); 基质金属蛋白酶(matrix metalloproteinase, MMP); 髓过氧化物酶 (myeloperoxidase, MPO); 嗜酸性粒细胞阳离子蛋白 (eosinophil cationic protein,ECP); 基质金属蛋白酶组织抑制因子 1(tissue inhibitor of matrix metalloproteinase 1,TIMP-1); 脱氧核糖核酸酶 I (deoxyribonuclease I, DNase I) ; 趋化因子 CXC 亚家族受体 (CXC subfamily receptor, CXCR)

支气管哮喘 (以下简称哮喘) 是由多种细胞 (包括气道的炎性细胞和结构细胞) 以及细胞组分 参与的气道慢性炎症性疾病, 临床表现为喘息、气 急、咳嗽等 ${ }^{[1]}$ 。以往认为哮喘主要由 2 型炎症因 子驱动, Th2 细胞分泌 IL-4、IL-5 和 IL-13, 引起气 道嗜酸性粒细胞炎症 ${ }^{[1]}$ 。随着研究的深人, 发 现 $50 \%$ 以上的哮喘存在以非嗜酸性粒细胞浸润为 主的气道炎症, 多以中性粒细胞性浸润为主 ${ }^{[2-3]}$ 。 中性粒细胞哮喘与持续性哮喘、重症哮喘、致死性 哮喘关系密切, 已逐渐引起临床医生和科研工作 者的关注和重视。中性粒细胞在骨髓中发育, 在 外周血中完全成熟, 其作为先天免疫防御的第一 道防线, 通过吞噬、脱颗粒、免疫应激发挥抗病原 体作用 ${ }^{[4]}$ 。然而目前的研究表明中性粒细胞的功 能远不止于此, 其还能分泌广泛的细胞因子、细胞 胞外诱捕网等效应分子, 参与先天和适应性免疫 反应, 其作用机制示意见图 1。因此, 中性粒细胞 在多种疾病的发病机制中起着至关重要的作用, 包括自身免疫性疾病、慢性炎症性疾病 (如哮喘) 等 ${ }^{[5]}$ 。本文就近年对中性粒细胞在哮喘中的作用 和地位研究进展作一综述。

\section{1 中性粒细胞哮喘的认识}

哮喘是一种异质性疾病, 可根据临床症状、特 异性诱因、治疗反应或炎症表型进行分类 ${ }^{[6]}$ 。由 过敏原刺激导致的以气道嗜酸性粒细胞炎症为基 础的哮喘可通过糖皮质激素或相应的 IL-4、IL-5 抗 体治疗, 但还有一部分哮喘患者并未显示出对这 些药物的治疗效应, 其气道炎症细胞浸润并非以 嗜酸性粒细胞为主, 目前将这一类型哮喘归为非 嗜酸性粒细胞哮喘。根据浸润细胞的比例, 非嗜 酸性粒细胞哮喘可进一步分为中性粒细胞哮喘、 混合粒细胞哮喘和寡细胞哮喘 ${ }^{[7-8]}$ 。中性粒细胞 哮喘目前还没有明确的定义。 Liu 等 ${ }^{[7]}$ 将中性粒 细胞哮喘描述为痰嗜酸性粒细胞小于 $2.5 \%$, 中性 粒细胞大于 $65 \%$; 而 Taylor 等 ${ }^{[9]}$ 则认为中性粒细 胞哮喘表型为痰液中存在 $61 \%$ 或更多的中性粒细 胞; 还有研究认为, 中性粒细胞哮喘的诊断存在年 龄差异,但其中性粒细胞均大于 $61 \%{ }^{[10]}$ 。除了诊断 标准, 中性粒细胞哮喘炎症细胞占比的稳定性也 存在争议。研究发现, 在两年的时间里, 只有 $8 \%$ 的 中性粒细胞哮喘患者炎症细胞占比保持了稳定, 而非嗜酸性粒细胞哮喘(包括中性粒细胞哮喘)不 


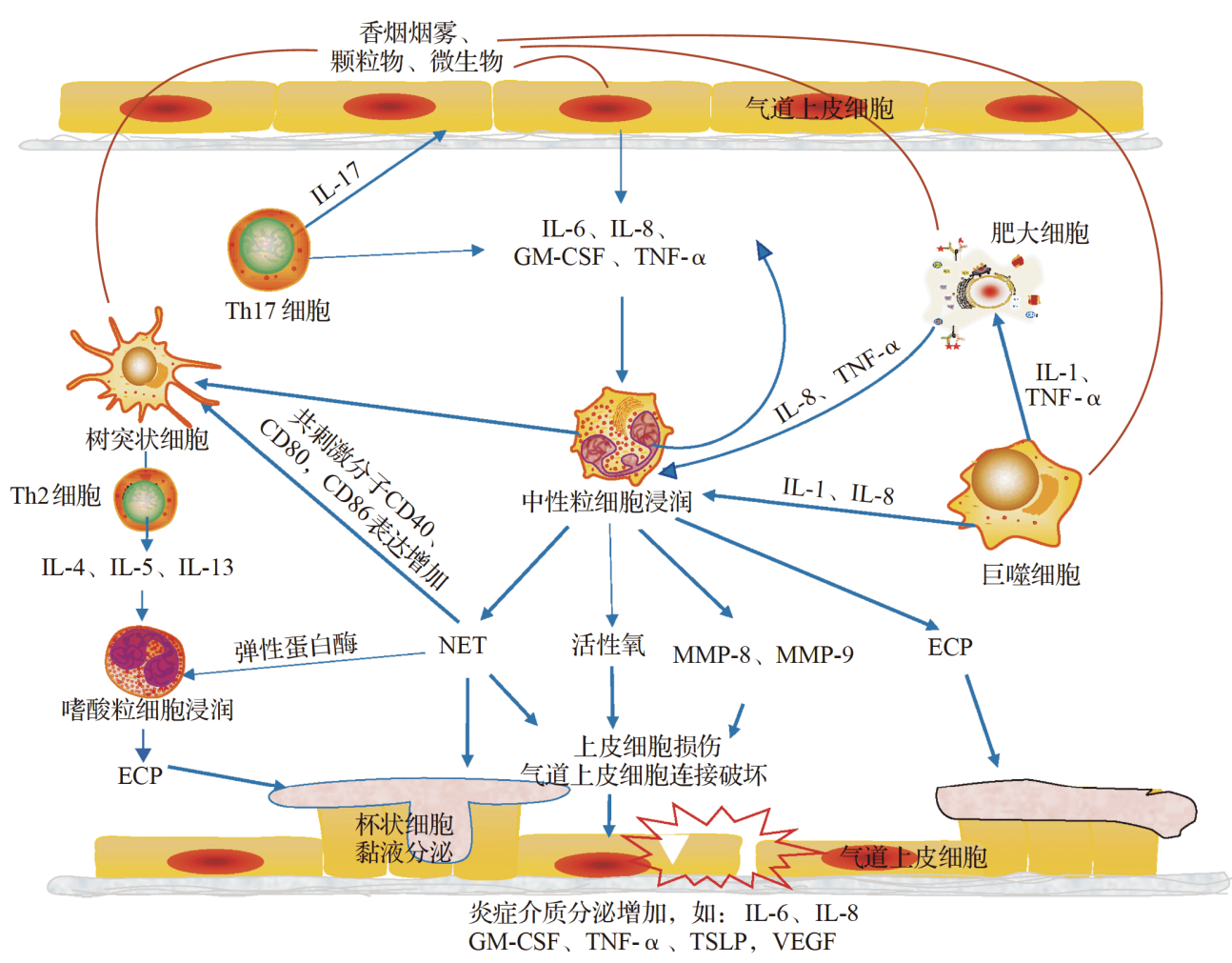

香烟烟雾、颗粒物、微生物等刺激气道上皮细胞、树突状细胞、巨噬细胞等产生 IL-6、IL-8、GM-CSF 等细胞因子,引起中性粒细胞趋化增 加及调亡下降; 浸润的中性粒细胞活化可产生活性氧、MMP、NET等,引起气道上皮损伤,损伤的气道上皮可进一步分泌趋化因子及炎症介 质等,加重气道炎症反应. IL: 白细胞介素;GM-CSF : 粒细胞-巨噬细胞集落刺激因子;TNF : 肿瘤坏死因子;NET: 中性粒细胞胞外诱捕网; MMP: 基质金属蛋白酶;ECP: 嗜酸性粒细胞阳离子蛋白;TSLP : 胸腺基质淋巴细胞生成素; VEGF:血管内皮生长因子.

图 1 中性粒细胞在哮喘中的作用机制示意图

Figure 1 Molecular mechanisms of neutrophils in asthma

管在短期(4周)还是长期 (5.3 年) 表型均稳定 ${ }^{[8,11]}$ 。

目前认为, 多种因素如烟草暴露 ${ }^{[12]}$ 、激素使 用 ${ }^{[13]}$ 、各种微生物感染 ${ }^{[14-16]}$ 、颗粒吸 ${ }^{[17-18]}$ 、臭氧 暴露 ${ }^{[19]}$ 均可使哮喘模型气道中性粒细胞增多。

\section{2 哮喘患者气道中性粒细胞增多的机制}

\section{1 中性粒细胞趋化增加}

中性粒细胞趋化需要趋化因子与受体结合, 并通过与血管识别、黏附、滚动、迁移等过程到达 趋化部位。哮喘患者的痰液和鼻腔分泌物中均可 检测到趋化因子 IL-8 水平升高 ${ }^{[20]}$, 中性粒细胞 在 IL-8 的趋化下利用选择素和整合素结合穿过内 皮细胞, 分泌蛋白酶穿透基底膜, 到达炎症部位, 参与气道炎症反应 ${ }^{[21]}$ 。除了 IL-8, 哮喘患者痰液 中 IL-17A 水平显著高于健康人群, 并且 IL-17A 水 平与痰液中性粒细胞计数呈正相关 ${ }^{[22]}$ 。动物实 验也发现, 经卵清蛋白及脂多糖刺激的小 鼠 Th17 细胞进人气道并释放 IL-17, 气道中性粒细 胞随之增加 ${ }^{[23]}$ 。IL-17A 可促进上皮细胞和内皮
细胞分泌细胞因子 (如 IL-6、GM-CSF、TNF- $\alpha$ 等)、 趋化因子 (IL-1、IL-8) 及炎症介质 (如一氧化氮 和 MMP 等), 促进中性粒细胞活化和募集。另外, Th17 还可直接产生并分泌趋化因子 IL-8, 促进中 性粒细胞活化和募集, 从而介导中性粒细胞引起 的炎症 ${ }^{[24-25]}$ 。

\section{2 中性粒细胞调亡减少}

多种机制共同导致哮喘中性粒细胞寿命延 长, 气道炎症加重。哮喘患者血浆、支气管肺泡灌 洗液或痰液中可检测到 IL-8、IL-17A、LTB4、GMCSF 和 TNF- $\alpha$ 等炎症介质浓度增加 ${ }^{[26-28]}$ 。GM-CSF 可通过细胞外调节蛋白激酶 $1 / 2$ 途径抑制中性粒 细胞凋亡 ${ }^{[29]}$ 。LTB4 通过与其相应的 LTB4 受体结 合, 下调环磷酸腺苷介导的中性粒细胞调亡 ${ }^{[30]}$ 。 除此之外,LTB4 还可通过活性氧依赖的促调亡蛋 白 Bad 降解来抑制中性粒细胞调亡, 其机制有赖 于核因子 $\kappa \mathrm{B}$ 信号通路的氧化还原调节 ${ }^{[31-32] 。}$ $\mathrm{S} 100 \mathrm{~A} 8$ 和 S100A9 是钙结合 S100 蛋白家族的重要 成员, 主要由中性粒细胞和单核细胞表达。哮喘小 
鼠或患者支气管肺泡灌洗液中 S100A8 和 S100A9 浓度较正常支气管肺泡灌洗液升高 ${ }^{[33-34]}$ 。经 S100A8 和 S100A9 刺激后的人支气管上皮细胞分 泌单核细胞趋化蛋白 $1 、$ IL- 6 和 IL-8, 产生的细胞 因子抑制半胱天冬酶 9 和半胱天冬酶 3 的激活, 使 哮喘的中性粒细胞调亡减少 ${ }^{[35]}$ 。

\section{3 中性粒细胞在哮喘起始阶段的作用}

哮喘小鼠气道中不仅存在中性粒细胞, 而且 其出现时间更早于嗜酸性粒细胞, 也就是说中性 粒细胞可能在哮喘起始阶段发挥作用。笔者团队 在以往研究中发现, 第一次卵清蛋白刺激之后, 中 性粒细胞从第 3 个小时开始增多, 而嗜酸性粒细 胞直到第 24 小时才开始增多 ${ }^{[36]}$ 。Toussaint 等 ${ }^{[37]}$ 也发现, 小鼠哮喘模型给予鼻病毒刺激后第 1 天可 见中性粒细胞明显增加, 而嗜酸性粒细胞无明显 变化。在低剂量脂多糖和屋尘螨诱导的小鼠哮喘 模型中, 中性粒细胞从第 6 小时开始增加, 且该群 中性粒细胞还可分泌 NET 诱导哮喘炎症 ${ }^{[16]}$ 。以 上研究均提示, 中性粒细胞作为血液中最丰富的 白细胞, 是先天免疫的第一道防线, 可能比嗜酸性 粒细胞更早地参与哮喘的发生。

机制研究还进一步证实了中性粒细胞在后续 哮喘炎症中的作用。Radermecker 等 ${ }^{[16]}$ 发现, 低剂 量脂多糖刺激后募集到气道的中性粒细胞可特异 性表达 CXCR4, 该群细胞可分泌 NET, 诱导哮喘炎 症和 Th2 反应。这可能与 NET 募集并激活树突状 细胞, 表面共刺激分子 CD40、CD80 和 CD86 表达 增加, 促进树突状细胞提呈抗原有关 ${ }^{[38]}$ 。在鼻病 毒诱导的小鼠哮喘模型中, 研究者发现来源 于 NET 的双链 DNA 介导鼻病毒诱导的过敏性哮 喘加重, 表现为气道炎症细胞浸润, 细胞因子分泌 增加, 䍄液分泌增多 ${ }^{[37]}$ 。后续研究也表明,通过 抑制中性粒细胞及其产生的物质, 可以减轻气道 炎症及哮喘的症状。如由脂多糖和屋尘螨诱导的 小鼠哮喘模型中, 中性粒细胞趋化因子受体 CXCR2 抗体、DNase I、弹性蛋白酶抑制剂等可减 轻小鼠气道炎症细胞浸润,使气道炎症评分及黏 液分泌下降, 细胞因子合成减少, 从而气道炎症减 轻 ${ }^{[16]}$ 。另一项研究也显示了类似的结果, 在由屋 尘螨及鼻病毒构建的哮喘急性加重模型中, 通 过 DNase I 降解 NET 可以有效缓解哮喘急性加重 小鼠的气道炎症 ${ }^{[37]}$ 。
上述研究均提示中性粒细胞可能比嗜酸性 粒细胞更早到达气道, 通过产生 NET, 分泌各种 酶、炎症介质、细胞因子等介导哮喘 Th2 炎症,引 起气道高反应性。中性粒细胞在哮喘早期炎症的 具体作用、机制和途径值得重视和进一步探索。

\section{4 中性粒细胞在哮喘效应阶段的作用}

中性粒细胞在哮喘的不同阶段分泌的物质不 同 ${ }^{[39]}$ 。早期主要分泌 MMP-9、弹性蛋白酶、氧自 由基和 MPO 等,晚期主要分泌 ECP 和 IL-8。

MMP-9 可由不同的细胞产生,但主要来源于 中性粒细胞 ${ }^{[39-40]}$, 可降解细胞外基质。中性粒细 胞哮喘患者体内总 MMP-9 水平非常高, 且与患者 肺功能呈负相关, 但 99\%以上的MMP-9不活跃, 存 在高水平的 MMP-9/TIMP-1 复合物 , MMP/TIMP-1 失衡参与了哮喘气道重塑 ${ }^{[40-41]}$ 。弹性蛋白酶参与 的哮喘病理生理过程包括上皮细胞损伤、支气管 黏液腺化生和气道高反应性。除此之外,弹性蛋 白酶还可诱导 IL-8 分泌, 促进中性粒细胞向肺募 集, 并诱导嗜酸细胞产生 $\mathrm{ECP}^{[39]}$ 。中性粒细胞还 能释放氧自由基, 通过直接与周边细胞分子发生 反应，或间接抑制抗蛋白酶活性等导致组织损 伤 ${ }^{[39]}$ 。除此之外,活性氧是一种关键的信号分 子, 刺激炎症因子产生, 参与过敏性气道炎症, 从 而引起气道高反应性 ${ }^{[42-43]}$ 。 Kim 等 ${ }^{[44]}$ 也指出活 性氧可用于评估中性粒细胞在严重哮喘中的功能 状态。研究表明, 哮喘患者与健康人群相 比 MPO 水平更高 ${ }^{[45]}$ 。分泌的 MPO 可以与呼吸爆 发时产生的过氧化氢反应产生次氯酸, 导致周边 组织损伤及炎症反应 ${ }^{[46]}$ 。MPO 还与中性粒细胞 弹性蛋白酶、双链 DNA 等参与构成 NET, 导致气 道上皮及血管内皮受损 ${ }^{[47-48]}$ 。IL-8 是中性粒细胞 强有力的趋化因子,气道上皮细胞、嗜酸性粒细胞 及中性粒细胞等多种细胞均可产生 IL-8 ${ }^{[39]}$, 早期 分泌的 MMP-9 和弹性蛋白酶可以促进气道上皮细 胞分泌 IL-8, 导致气道持续的中性粒细胞趋化的 恶性循环 ${ }^{[13]}$ 。IL-8 除了引起气道炎症外, 还可通 过促进气道平滑肌细胞的增殖及延长其寿命来增 加气道平滑肌细胞的数量, 从而加重气道重塑及 气道高反应性 ${ }^{[49-50]}$ 。

\section{5 以中性粒细胞为靶点的哮喘治疗方法}

IL-17 细胞因子通过与 IL-17 受体结合发挥趋 
化中性粒细胞的作用 ${ }^{[51]}$ 。IL-17 抗体可减轻小鼠 哮喘模型中的气道炎症浸润和气道高反应 性 ${ }^{[52]}$ 。哮喘合并感染患者进行抗 IL-17 单克隆抗 体治疗后, 支气管肺泡灌洗液中性粒细胞数量显 著减少 ${ }^{[24]}$ 。但另一项临床试验研究显示, 中重度 哮喘患者使用抗 IL-17A 受体抗体并未显示出期望 的治疗效果 ${ }^{[53]}$ 。一项 IL-13 和 IL-17 的双特异性 抗体 BITS7201A 临床试验结果显示, 该药物的安 全性和耐受性可,但其抗体形成率高, 可能影响治 疗效果, 并且可能增加过敏反应 ${ }^{[54]}$ 。因此, 对 于 IL-17 相关信号通路在哮喘治疗中的应用还需 要进一步研究。

CXCR2 是 IL-8 的高亲和力受体,SCH 527123 是一种新型的小分子, 可同时阻断CXCR1和CXCR2, 降低哮喘受试患者气道中性粒细胞的水平, 但其 治疗效果仍未明确 ${ }^{[55]}$ 。另一项研究结果也表明, 使用SCH527123 可减少哮喘患者痰液中约 $37 \%$ 的 中性粒细胞, 但并未体现更好的哮喘控制及肺功 能改善等作用 ${ }^{[56]}$ 。其他CXCR2拮抗剂如AZD5069 也可以减少痰液中 $90 \%$ 的中性粒细胞数量, 但另 一项B 期临床试验并未提示这一药物可以改善临 床结果 ${ }^{[57-58]}$ 。

大环内酯类药物不仅具有抗菌功能, 还可以 发挥抗炎作用 ${ }^{[59]}$ 。大环内酯类药物通过抑制气 道上皮细胞核因子 $\kappa \mathrm{B}$ 受体抑制 IL- 8 释放, 以及抑 制磷脂酰肌醇 3-激酶等途径发挥作用, 抑制气道 炎症 ${ }^{[10,60]}$ 。在小鼠哮喘模型中, 克拉霓素协同地 塞米松能更有效地下调气道阻力, 减轻气道炎症 细胞浸润, 缓解哮喘症状 ${ }^{[59]}$ 。在严重难治性非嗜 酸性粒细胞哮喘患者中, 克拉霉素治疗可降低主

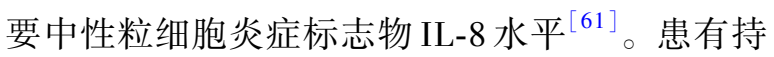
续症状性哮喘的受试者在口服阿奇霉素治 疗 48 周后哮喘发作较少, 生活质量改善, 但这种 疗法的长期影响还需要进一步评估 ${ }^{[62]}$ 。总体来 说, 大环内酯类药物对哮喘有改善作用, 但是否可 纳人中性粒细胞哮喘的常规治疗, 及其用药指征 和用量等还需要更多的临床证据支持。

NET 具有抗菌功能, 但其过量存在可导致肺 组织不同程度损伤。动物实验表明, 通过降解 NET 的成分及结构可以减轻哮喘小鼠的气道炎症 和细胞因子分泌, 同时减轻哮喘小鼠急性加重的 炎症表现 ${ }^{[16,37]}$ 。未来 NET 有望用于哮喘的预防 及哮喘加重的治疗中。

\section{6 结 语}

中性粒细胞已引起临床医生和科研工作者的 重视, 但中性粒细胞在哮喘中的作用尚存在许多 疑问,其发病机制尚未完全清楚。未来期待更多 阐述中性粒细胞在哮喘中的研究面世, 尤其是中 性粒细胞哮喘的诊断标准、浸润到肺组织的中性 粒细胞亚型、中性粒细胞在哮喘早期炎症的介导 作用等。这些将有助于获得准确的流行病学资 料、推动高质量的中性粒细胞哮喘临床研究, 并为 中性粒细胞哮喘的治疗提供更多思路和精准化治 疗的方向。

\section{参考文献}

[1] PETERS M C, WENZEL S E. Intersection of biology and therapeutics:type 2 targeted therapeutics for adult asthma $[\mathrm{J}]$. Lancet, 2020,395(10221):371-383. DOI: 10. 1016/S0140-6736(19)33005-3.

[2] ZHANG J, ZHU Z, ZUO X, et al. The role of NTHi colonization and infection in the pathogenesis of neutrophilic asthma [J]. Respir Res, 2020,21 (1) : 170. DOI: $10.1186 / \mathrm{s} 12931-020-01438-5$.

[3] GRUNWELL J R, STEPHENSON S T, TIROUVANZIAM R, et al. Children with neutrophil-predominant severe asthma have proinflammatory neutrophils with enhanced survival and impaired clearance $[\mathbf{J}]$. J Allergy Clin Immunol-Practice, 2019,7(2):516525. e6. DOI: 10. 1016/j. jaip. 2018. 08. 024.

[4] KHAN M A, ALI Z S, SWEEZEY N, et al. Progression of cystic fibrosis lung disease from childhood to adulthood:neutrophils, neutrophil extracellular trap (NET) formation, and net degradation $[\mathrm{J}]$. Genes, 2019,10(3): 183. DOI: 10. 3390/genes 10030183 .

[5] MANTOVANI A, CASSATELLA M A, COSTANTINI C, et al. Neutrophils in the activation and regulation of innate and adaptive immunity $[\mathrm{J}]$. Nat Rev Immunol, 2011,11(8):519-531. DOI: 10. 1038/nri3024.

[6] RADERMECKER C, LOUIS R, BUREAU F, et al. Role of neutrophils in allergic asthma $[\mathrm{J}]$. Curr Opin Immunol, 2018, 54:28-34. DOl:10. 1016/j. coi. 2018. 05. 006.

[7] LIU W, CHEN H, ZHANG D, et al. A retrospective study of clinical features of cough variant asthma in Chinese adults $[\mathrm{J}]$. Allergy Asthma Clin Immunol, 2019,15(1):3. DOI:10. 1186/s13223-019-0318-5

[8 ] SIMPSON J L, SCOTT R, BOYLE M J, et al. Inflammatory subtypes in asthma: Assessment and identification using induced sputum $[J]$. Respirology, 2006,11(1) : 54-61. DOI: 10. 1111/j. 1440-1843. 2006. 00784. x.

[9] TAYLOR S L, LEONG L E X, CHOO J M, et al. Inflam- 
matory phenotypes in patients with severe asthma are associated with distinct airway microbiology $[\mathbf{J}]$. J Allergy Clin Immunol , 2018, 141 (1) :94-103. e115. DOl: 10. 1016/j. jaci. 2017. 03. 044.

[10] NAIR P, PRABHAVALKAR K S. Neutrophilic asthma and potentially related target therapies $[\mathrm{J}]$. Curr Drug Targets, 2020,21(4):374-388. DOI: 10. $2174 / 1389450120666191011162526$.

[11] NAIR P, AZIZ-UR-REHMAN A, RADFORD K. Therapeutic implications of 'neutrophilic asthma' [J]. Curr Opin Pulmonary Med, 2015,21(1): 33-38. DOI : 10. 1097/MCP. 0000000000000120.

[12] PANETTIERI JR. R A. Neutrophilic and pauciimmune phenotypes in severe asthma $[\mathrm{J}]$. Immunol Allergy Clinics North Am, 2016,36(3):569-579. DOI : 10. 1016/j. iac. 2016. 03. 007.

[13] SEYS S F, LOKWANI R, SIMPSON J L, et al. New insights in neutrophilic asthma $[\mathrm{J}]$. Curr Opin Pulmonary Med, 2019,25(1):113-120. DOI: 10 . 1097/MCP. 0000000000000543.

[14] PATEL K K, WEBLEY W C. Respiratory chlamydia infection induce release of hepoxilin A3 and histamine production by airway neutrophils $[\mathrm{J}]$. Front Immunol, 20189. DOI : 10. 3389/fimmu. 2018. 02357.

[15] WANG G, PANG Z, CHEN-YU HSU A, et al. Combined treatment with SB203580 and dexamethasone suppresses non-typeable Haemophilus influenzaeinduced Th17 inflammation response in murine allergic asthma $[J]$. Eur J Pharmacol, 2019,862: 172623. DOI: 10. 1016/j. ejphar. 2019. 172623.

[16] RADERMECKER C, SABATEL C, VANWINGE C, et al. Locally instructed CXCR4hi neutrophils trigger environment-driven allergic asthma through the release of neutrophil extracellular traps $[J]$. Nat Immunol, 2019,20(11) : 1444-1455. DOI : 10. 1038/ s41590-019-0496-9.

[17] GENG X, WANG X, LUO M, et al. Induction of neutrophil apoptosis by a $\mathrm{Bcl}-2$ inhibitor reduces particulate matter-induced lung inflammation $[\mathrm{J}]$. Aging, 2018,10(6): 1415-1423. DOI : 10. 18632/ aging. 101477.

[18] PANG L,ZOU S,SHI Y, et al. Apigenin attenuates PM2. 5-induced airway hyperresponsiveness and inflammation by down-regulating $\mathrm{NF}-\kappa \mathrm{B}$ in murine model of asthma[J]. Int J Clin Exp Pathol , 2019, 12 (10):3700-3709.

[19] SHORE S A. Mechanistic basis for obesity-related increases in ozone-induced airway hyperresponsiveness in mice[J]. Ann ATS, 2017,14(Supplement_5 ):S357-S362. DOI:10.1513/AnnalsATS. 201702-140AW.

[20] RAY A, KOLLS J K. Neutrophilic inflammation in asthma and association with disease severity $[\mathrm{J}]$.
Trends Immunol, 2017,38(12) :942-954. DOI : 10. 1016/j. it. 2017.07. 003.

[21] MATSUSHIMA H, GENG S, LU R, et al. Neutrophil differentiation into a unique hybrid population exhibiting dual phenotype and functionality of neutrophils and dendritic cells $[\mathrm{J}]$. Blood, 2013,121 (10) : 1677-1689. DOI : 10. 1182/blood-2012-07445189.

[22] ZHAN C, XU R, LIU J, et al. Increased sputum IL17A level in non-asthmatic eosinophilic bronchitis [J]. Lung, 2018,196(6) :699-705. DOI : 10. 1007/ s00408-018-0166-y.

[23] WHITEHEAD G S,KANG H S, THOMAS S Y, et al. Therapeutic suppression of pulmonary neutrophilia and allergic airway hyperresponsiveness by a ROR $\gamma \mathrm{t}$ inverse agonist [J/OL]. JCI Insight, 2019,5(14): e125528. DOl:10. 1172/jci. insight. 125528.

[24] WITOWSKI J, KSIEK K, JÖRRES A. Interleukin-17 : a mediator of inflammatory responses $[\mathrm{J}]$. Cell Mol Life Sci, 2004,61(5):567-579. DOI: 10. 1007/ s00018-003-3228-z.

[25] LUCHERINI O M, LOPALCO G, CANTARINI L, et al. Critical regulation of Th17 cell differentiation by serum amyloid-A signalling in Behcet's disease $[\mathrm{J}]$. Immunol Lett, 2018,201:38-44. DOI : 10. 1016/j. imlet. 2018. 10. 013.

[26] DIMITROVA D, YOUROUKOVA V, IVANOVATODOROVA E, et al. Serum levels of IL-5, IL-6, IL8, IL-13 and IL-17A in pre-defined groups of adult patients with moderate and severe bronchial asthma [J]. Respiratory Med, 2019,154:144-154. DOI : 10. 1016/j. rmed. 2019. 06. 024.

[27] PAL K, FENG X, STEINKE J W, et al. Leukotriene A4 hydrolase activation and leukotriene B4 production by eosinophils in severe asthma $[\mathrm{J}]$. Am J Respir Cell Mol Biol, 2019,60(4):413-419. DOI : 10 . 1165/rcmb. 2018-01750C.

[28] ELLER M C N, VERGANI K P, SARAIVAROMANHOLO B M, et al. Can inflammatory markers in induced sputum be used to detect phenotypes and endotypes of pediatric severe therapyresistant asthma? $[\mathrm{J}]$. Pediatr Pulmonol, 2018,53 (9) : 1208-1217. DOI: 10. 1002/ppul. 24075.

[29] PINTARD C, BEN KHEMIS M, LIU D, et al. Apocynin prevents GM-CSF-induced-ERK $1 / 2$ activation and -neutrophil survival independently of its inhibitory effect on the phagocyte NADPH oxidase NOX2 $[\mathrm{J}]$. Biochem Pharmacol, 2020,177: 113950. DOI : 10. 1016/j. bcp. 2020. 113950.

[30 ] HILLIARD K A, BLAHO V A, JACKSON C D, et al. Leukotriene B4 receptor BLT1 signaling is critical for neutrophil apoptosis and resolution of experimental Lyme arthritis[J]. FASEB J, 2020,34(2) :2840- 
2852. DOI: $10.1096 /$ fj. 201902014R.

[31] MCCRACKEN J M, ALLEN L A H. Regulation of human neutrophil apoptosis and lifespan in health and disease $[\mathrm{J}]$. J Cell Death, 2014,7:JCD. S11038. DOI: $10.4137 / J C D$. S1 1038.

[32] BARCELLOS-DE-SOUZA P, CANETTI C,BARJAFIDALGO C, et al. Leukotriene B(4) inhibits neutrophil apoptosis via NADPH oxidase activity: redox control of NF- $\kappa \mathrm{B}$ pathway and mitochondrial stability $[\mathrm{J}]$. Biochim Biophys Acta, 2012,1823 (10): 1990-1997. DOl:10. 1016/j. bbamcr. 2012. 07.012.

[33] 顾晓菲, 陈金金沝, 陈慧君, 等. S100A8/RAGE、 Caveolin-1 在中性粒细胞性支气管哮喘大鼠中的 作用及罗红霉素对其表达的影响 $[\mathrm{J}]$. 中华结核和 呼吸杂志,2019,42(11):845-851. DOI：10.3760/ cma. j. issn. 1001-0939. 2019. 11. 012.

GU Xiaofei,CHEN Xinmiao, CHEN Huijun, et al. The role of S100A8/RAGE and Caveolin-1 and the effect of roxithromycin on their expression in a rat model of neutrophilic asthma $[\mathrm{J}]$. Chinese Journal of Tuberculosis and Respiratory Diseases, 2019, 42(11):845-851. DOI: $10.3760 / \mathrm{cma}$. j. issn. 10010939. 2019. 11.012. (in Chinese)

[34] KIM D H, CHOI E, LEE J S, et al. House dust mite allergen regulates constitutive apoptosis of normal and asthmatic neutrophils via toll-like receptor $4[\mathrm{~J} / \mathrm{OL}]$. PLoS One, 2015,10(5):e0125983. DOI: 10. 1371/ journal. pone. 0125983

[35] KIM D H, GU A, LEE J S, et al. Suppressive effects of S100A8 and S100A9 on neutrophil apoptosis by cytokine release of human bronchial epithelial cells in asthma [J]. Int J Med Sci, 2020,17(4) :498-509. DOI: $10.7150 /$ ijms. 37833.

[36] WENG Q, ZHU C, ZHENG K, et al. Early recruited neutrophils promote asthmatic inflammation exacerbation by release of neutrophil elastase $[\mathrm{J}]$. Cellular Immunol, 2020,352:104101. DOI: 10. 1016/j. cellimm. 2020. 104101.

[37] TOUSSAINT M,JACKSON D J,SWIEBODA D, et al. Host DNA released by NETosis promotes rhinovirusinduced type-2 allergic asthma exacerbation $[\mathrm{J}]$. Nat Med, 2017,23(6):681-691. DO1:10. 1038/nm. 4332 .

[38 ] WEERAPPULI P D, LOUTTIT C, KOJIMA T, et al. Extracellular trap-mimicking DNA-histone mesostructures synergistically activate dendritic cells $[\mathrm{J} / \mathrm{OL}]$. Adv Healthcare Mater, 2019,8(22) : 1900926. DOI: 10. 1002/adhm. 201900926.

[39] MONTESEIRÍN J. Neutrophils and asthma $[J]$. J Investig Allergol Clin Immunol, 2009, 19(5) :340354.

[40] GRZELA K, LITWINIUK M, ZAGORSKA W, et al.
Airway remodeling in chronic obstructive pulmonary disease and asthma:the role of matrix metalloproteinase-9[J]. Arch Immunol Ther Exp, 2016,64 (1) :47-55. DOI: $10.1007 / \mathrm{s} 00005-015-0345-\mathrm{y}$.

[41] SIMPSON J L, SCOTT R J, BOYLE M J, et al. Differential proteolytic enzyme activity in eosinophilic and neutrophilic asthma $[\mathbf{J}]$. Am J Respir Crit Care Med, 2005,172(5):559-565. DOI: 10. 1164/rccm. 200503-3690C.

[42] CUI H, HUANG J, LU M, et al. Antagonistic effect of vitamin $\mathrm{E}$ on nAl2O3-induced exacerbation of Th2 and Th17-mediated allergic asthma via oxidative stress [J]. Environ Pollut, 2019,252:1519-1531. DOI : 10. 1016/j. envpol. 2019. 06. 092.

[43] LI Y, ZHANG L, WANG X, et al. Effect of Syringic acid on antioxidant biomarkers and associated inflammatory markers in mice model of asthma $[\mathrm{J}]$. Drug Dev Res, 2019,80(2):253-261. DOI:10. 1002/ ddr. 21487.

[44] KIM S H, UUGANBAYAR U, TRINH H K T, et al. Evaluation of neutrophil activation status according to the phenotypes of adult asthma $[\mathrm{J}]$. Allergy Asthma Immunol Res, 2019,11(3):381. DOI:10.4168/ aair. 2019. 11.3.381.

[45] KEATINGS V M, BARNES P J. Granulocyte activation markers in induced sputum:comparison between chronic obstructive pulmonary disease, asthma, and normal subjects $[\mathbf{J}]$. Am J Respir Crit Care Med, 1997,155(2):449-453. DOI: 10 . 1164/ajrccm. 155. 2. 9032177.

[46] ALI I, KHAN S N, CHATZICHARALAMPOUS C, et al. Catalase prevents myeloperoxidase self-destruction in response to oxidative stress $[\mathrm{J}]$. J Inorg Biochem, 2019,197:110706. DOI:10. 1016/j. jinorgbio. 2019. 110706.

[47] MOSCHONAS I C, TSELEPIS A D. The pathway of neutrophil extracellular traps towards atherosclerosis and thrombosis $[\mathrm{J}]$. Atherosclerosis, 2019,288:916. DOI : 10. 1016/j. atherosclerosis. 2019. 06. 919.

[48] LACHOWICZ-SCROGGINS M E, DUNICAN E M, CHARBIT A R, et al. Extracellular DNA, neutrophil extracellular traps, and inflammasome activation in severe asthma $[$ J]. Am J Respir Crit Care Med, 2019,199(9):1076-1085. DOI : 10. 1164/recm. 20181018690C.

[49] MURCIA R Y, VARGAS A, LAVOIE J P. The interleukin-17 induced activation and increased survival of equine neutrophils is insensitive to glucocorticoids [J/OL]. PLoS One, 2016,11(5): e0154755. DOI: 10. 1371/journal. pone. 0154755.

[50］张星慧, 常晓悦. 中性粒细胞性哮喘与其相关细胞 因子 $[\mathrm{J}]$. 国际呼吸杂志, 2017,37(23): 1815-1818. DOI: 10. 3760/cma. j. issn. 1673-436X. 2017. 23. 011. 
ZHANG Xinghui,CHANG Xiaoyue. Neutrophilic asthma and other cytokines $[\mathrm{J}]$. International Journal of Respiration, 2017,37(23) : 1815-1818. DOI: $10.3760 / \mathrm{cma}$. j. issn. 1673-436X. 2017. 23. 011. (in Chinese)

[51] RODRÍGUEZ-CERDEIRA C,GONZÁLEZ-CESPÓN J L,MARTÍNEZ-HERRERA E, et al. Candida infections in patients with psoriasis and psoriatic arthritis treated with interleukin-17 inhibitors and their practical management $[\mathrm{J}]$. G Ital Dermatol Venereol, 2020. DOI: 10. 23736/S0392-0488. 20. 06580-3

[52] LIANG L, HUR J, KANG J Y, et al. Effect of the antiIL-17 antibody on allergic inflammation in an obesityrelated asthma model $[\mathrm{J}]$. Korean J Intern Med, 2018,33(6):1210-1223. DOI: 10. 3904/kjim. 2017. 207.

[53] BUSSE W W, HOLGATE S, KERWIN E, et al. Randomized, double-blind, placebo-controlled study of brodalumab,a human anti-IL-17 receptor monoclonal antibody, in moderate to severe asthma [J]. Am J Respir Crit Care Med, 2013,188(11): 1294-1302. DOI : 10. 1164/rccm. 201212-23180C.

[54] STATON T L, PENG K, OWEN R, et al. A phase I, randomized, observer-blinded, single and multiple ascending-dose study to investigate the safety, pharmacokinetics, and immunogenicity of BITS7201A,a bispecific antibody targeting IL-13 and IL-17, in healthy volunteers[J]. BMC Pulm Med, 2019,19 (1) :5. DOI: $10.1186 / \mathrm{s} 12890-018-0763-9$.

[55] TODD C M, SALTER B M, MURPHY D M, et al. The effects of a CXCR1/CXCR2 antagonist on neutrophil migration in mild atopic asthmatic subjects $[\mathrm{J}]$. Pulm Pharmacol Ther, 2016,41: 34-39. DOI: 10. 1016/ j. pupt. 2016. 09. 005.
[56] NAIR P,GAGA M,ZERVAS E, et al. Safety and efficacy of a CXCR2 antagonist in patients with severe asthma and sputum neutrophils: a randomized, placebo-controlled clinical trial $[\mathrm{J}]$. Clin Exp Allergy ,2012,42(7):1097-1103. DOl:10. 1111/ j. 1365-2222. 2012. 04014. x.

[57] WATZ H,UDDIN M,PEDERSEN F, et al. Effects of the CXCR2 antagonist AZD5069 on lung neutrophil recruitment in asthma $[\mathrm{J}]$. Pulm Pharmacol Ther, 2017,45:121-123. DOl:10. 1016/j. pupt. 2017. 05. 012.

[58] O'BYRNE P M, METEV H, PUU M, et al. Efficacy and safety of a CXCR2 antagonist,AZD5069, in patients with uncontrolled persistent asthma:a randomised, double-blind, placebo-controlled trial [J]. Lancet Respiratory Med, 2016,4(10):797806. DOI : 10. 1016/S2213-2600(16)30227-2.

[59] AN T J, RHEE C K, KIM J H, et al. Effects of macrolide and corticosteroid in neutrophilic asthma mouse model $[\mathrm{J}]$. Tuberc Respir Dis, 2018,81 (1) : 80. DOI: $10.4046 /$ trd. 2017. 0108.

[60] PANETTIERI R A JR. The role of neutrophils in asthma $[\mathrm{J}]$. Immunol Allergy Clin North Am, 2018,38(4):629-638. DOl:10. 1016/j. iac. 2018. 06.005.

[61] LOVERDOS K, BELLOS G, KOKOLATOU L, et al. Lung microbiome in asthma: current perspectives $[\mathrm{J}]$. JCM, 2019,8(11) : 1967. DOI : 10. 3390/jcm8111967.

[62 ] GIBSON P G, YANG I A, UPHAM J W, et al. Effect of azithromycin on asthma exacerbations and quality of life in adults with persistent uncontrolled asthma (AMAZES) : a randomised, double-blind, placebocontrolled trial $[\mathrm{J}]$. Lancet, 2017,390(10095):659668. DOI: 10. 1016/S0140-6736(17)31281-3.

[本文编辑 沈 敏 刘丽娜] 